\title{
A Thorough Investigation on Speech Enhancement Techniques for Hearing Aids
}

\author{
N. Shanmugapriya \\ Assistant Professor \\ Department of Computer Applications(MCA), \\ Dr. SNS Rajalakshmi College of Arts and Science, \\ Coimbatore -641 049, Tamil Nadu, India
}

\author{
E. Chandra, PhD \\ Director, Department of Computer \\ Applications(MCA), Dr. SNS Rajalakshmi College of \\ Arts and Science, \\ Coimbatore-641 049, Tamil Nadu, India
}

\begin{abstract}
In recent years, speech development has become an interesting area in the field of signal processing especially in the applications of hearing aids. In hearing aid applications, the speech enhancement technique has been employed mainly for dipping the additive background noise. During the speech enhancement process, high background noise occurs due to the rapture nature of the speech. In order to overcome this problem, various methods have been employed for increasing the speech quality of hearing aid application. This paper provides an outlook of the speech enhancement techniques in a detailed manner with various speech enhancement algorithms such as Wiener filtering, beamformer, generalized singular value decomposition, transformations, and spectral subtraction technique. These methods have been designed with the main aim of reducing background noise. This paper discussed the merits and demerits of all speech enhancement algorithms with their unique characteristic features. The performance of the algorithm has been evaluated with the help of parameter metrics such as PSEQ and SNR.
\end{abstract}

\section{Keywords}

Speech enhancement, Hearing Aids, Wiener filter, Spectral subtraction technique

\section{INTRODUCTION}

In recent decades, the hearing problems among people around the world have increased. It is a fact that people with hearing deficiency struggle to recognize necessary content from the original speech with noise due to interfering sounds, background noise and echo sounds. So, hearing aids have been widely employed to deal with this problem. Usual hearing aids have automatic volume control and frequencyselective amplification for resolving the hearing loss. Moreover, the directional microphones and filtering techniques are available for reducing the noise. In digital hearing aids, signal processing mainly involves multichannel dynamic range compression with selectable channels and settable values of attack time, release time, and compression ratio which is explained in [1].

Sensorineural deficiency is commonly connected with improved spectral masking because of widening auditory filters. In the past decades, the binaural dichotic presentation [2], spectral contrast enhancement [3], and multiband frequency compression [4] algorithm were proposed for reducing the spectral masking but these techniques were observed to have problems with speech perception. After this, the redundant signal energy is minimized in speech segregation algorithm for attaining the fine quality of a desired speech signal. In modern hearing aids, multiple microphones integrated with wireless binaural data linkage between left and right devices are employed for reducing the noise.

But modern devices have drawback in their ability of hearing the speech in the high frequency range. In order to resolve this problem, speech enhancement or noise reduction techniques are utilized in modern hearing aids. The classification of speech enhancement methods or noise reduction methods mainly depends on single and multiple microphone methods. The multi-microphone algorithm is a combination of beam former algorithm and single channel noise reduction algorithm that perform spatial filtering with spectro temporal filtering. The multi-microphone algorithm has the limitation that it needs high storage space computational and hardware complexity too.

Even though multi-microphone has certain drawbacks, it has better result than the single-microphone methods. But in modern hearing aids, the speech perception in noisy environments is very difficult. The cochlear prostheses and other sensory aid having the same drawback that is explained in [5]. In order to solve this problem, the second microphones are employed for referencing input in noise containment. But it is unfeasible when implementing for the real time application. In this work, various existing speech enhancement methods for hearing aids are conversed briefly with their advantages and disadvantages.

\section{SURVEY OF SPEECH ENHANCEMENT FOR HEARING AIDS}

Junfeng Li et al (2009) [6] presented a two-stage binaural speech enhancement based on the EC model with Wiener filter (TS-BASE/WF). In this TS-BASE/WF method, the target signals are balanced and abandoned (EC model) for evaluating the interfering signals and next targeted signals are enhanced through the use of a time-variant Wiener filter based on producing noisy mixture signals. The main advantages of the TS-BASE/WF are: (1) non-stationary multiple-source intrusive signals are proficiently preceded in this method (2) later than processing the speech files are divided simply. As wiener filter having limitation such as signal components coming from diverse directions and it reduce the overall performance of this method.

Santosh et al (2013) [7] proposed a spectral subtraction technique is in modern hearing aids utilized for real-time speech enhancement. In this method cascaded-median method is primarily exercised by author for evaluation of the noise spectrum and it decreases the computational complexity and memory constraint exclusion of voice activity recognition. 
Here the stationary and non stationary additive noise utilized for increasing the speech quality by increasing the SNR value. The main limitation of this method is the residual noise. In digital hearing aids for binaural speech enhancement systems, a beamformer is proposed by Heinrich and Peter Vary (2012) in [8].

In this method, the single module is primarily proposed for the evaluation of the Time-Difference-Of-Arrival (TODA) and time-alignment operates in the frequency domain. This has the positive point that this method has a low computational complexity. In order to attain the efficient evaluation of TODA, the cross-correlation along with phase transform weighting is proposed. Histogram based TODA is proposed to overcome the delay constraints. The timealignment is evaluated through the product of spectral phase factors. The beamformers is utilized to estimate the density of interrupted the source signal. The main limitation of this method is low frequency, microphone noise components and it affects the framework during the real time implementation as well.

Tim Van et al (2007) [9] described the binaural cue preservation of a noise reduction algorithm for bilateral hearing aids. The researcher utilized multichannel Wiener filter along with interaural transfer function extension (MWFITF). An additional term is included in the cost function to defend the binaural cues of both the speech and noise component of a signal in addition with noise reduction. The theoretical analysis is together with objective binaural performance evaluations and a perceptual evaluation for getting the successful results with this method. MWF-ITF needs the binaural unmasking cause for different speech and noise ITFs on a single frequency case of a speech quality improvement.

However, this binaural unmasking effect is not possible in MWF-ITF which is the main limitation of this method. Ulrik Kjems et al (2012) [10] proposed a maximum likelihood method for evaluating noise imposing on the microphone array. Even though the target speech signal is present in the environment this method permits prediction of noise matrix for non-stationary for non-stationary noise sources. In this approach, the noise matrix is calculated by estimating the power spectral density of the noise present in the SC filter. The major drawback of this method is an estimation of covariance matrix can be closely partial for small samples due to maximum likelihood.

John Woodruff et al (2011) [11] proposed a technique for calculating noise and echo in hearing aids. The single and multi-channel linear filters are utilized for diminishing noise and echo in hearing aid application. The author differentiates conservative and aggressive strategies. The previous methods majorly exercised for attaining a quick outcome from a postfilter of a beamformer. But, directionality based speech enhancement patterns also have two major drawbacks in other environments are: 1) the directional speech enhancement pattern has less performance than non-directional pattern since directional porter had more noise. The hearing aid with directional speech enhancement pattern is highly sensitive to wind noise than a non-directional pattern.

Heinrich and Peter (2009) et al [12] presented a new system for single-channel speech enhancements. The method is utilized to speech with low echo and very low background noise with a low signal delay and computational complexity. The generalized spectral subtraction rule is utilized in this method and it is merely based on the variation of the echo and background noise. The maximum likelihood is proposed to estimate the reverberation time (RT). This RT is utilized for estimating the spectral variation of speech. Based on the calculation of domain filtering with coefficient the speech quality is increased that is adapted in the non-uniform frequency domain.

The major drawback of this method is an evaluation of echo time can be highly partial for small samples due to maximum likelihood. The time domain filter is utilized to attain high speech quality, however the constructing a high quality filter is much difficult. This is the main limitation of time domain filter and another one is filter endures from the ripple. Klaus et al (2010) [13] explored a class of Blind Source Separation (BSS) based speech enhancement methods for binaural hearing aids. Here, the author investigated Blind Source Separation techniques for diverse scenarios. The number of sources should not exceed the number of sensors when the method is determined scenarios. When it is undetermined scenarios, then the number of active source signals more than a microphone.

The BSS algorithm is an unconventional to beam former algorithm as no a-prior knowledge of the sensor positions is needed. The merits of BSS are the optimization of the outcomes. This is majorly depends on mutual statistical of diverse source signals. Blind Source Separation is highly complicated to solve and computationally more expensive. JEON Yu-Yong et al (2011) [14] proposed a method to improve the speech quality that is reduced by environmental noise. The author proposed minima controlled recursive averaging (MCRA) algorithm for calculating the noise spectrum and the partial marking effect. Here he also introduced psychoacoustic features for reinforcing speech. The parameters exercised to estimate the performance of the existing method is PESQ (perceptual evaluation of speech quality) and segSNR (segmental signal to noise ratio).

Young et al (2007) [15] proposed a modified spectral subtraction and companding for increasing the speech quality in digital hearing aids. Here the noise level is completely reduced through the adjustment of the biases of the evaluated noise spectrum that depends on the substraction factor. The channel format is improved by means of speech indicator via implementing the companding. In this method the weak speech components are extracted during that extraction the noise level is diminished. The algorithm is experimented based on a variety of objective and subjective evaluation. The performance metrics utilized in this paper for evaluating the algorithm is SNR and log likelihood ratio. The Likelihood Ratio (LLR) has a less correlation and SNR ha the more correlation with subjective appraises

David et al (2013) [16] proposed a novel sound source separation technique or binaural speech enhancement depends on the power restrictions that including the equally computational cost and the transmission bit rate. This algorithm depends on supervised machine learning and timefrequency masking. The author utilized a tailored evolutionary algorithm for optimizing the transmission technique. It is mostly exercised for allocating the number of bits in the frequency band. Simon Doclo et al (2002) [17] proposed generalized singular value decomposition (GSVD) algorithm to enhance the microphone speech signals affected by additive colored noise. The author has improved single microphone signal algorithm for multi microphone signals 
This method majorly utilized for diminishing the noise in the source signal through GSVD and it addresses the specific optimal filtering problem when the desired response signal cannot be examined. Apte et al (2010) [18] proposed a method for background noise minimization. The magnitude of STFT of noisy speech is kept default, whereas the phase is modified by using this method. The modified phase spectrum and constant magnitude spectrum are combined to facilitate attaining complex spectra of speech. This modification outcomes leads to termination of low energy components of complex spectra. This is the major cause for minimizing background noise that is essential in hearing aids. SNRPerception models for AWGN, Train noise, and Babble noise are designed via Nonlinear Regression modeling technique.

This algorithm produces better speech quality for hearing aids through suppressing the background noise. However the main limitation that it includes very narrow peaks in speech spectrum ensuing in overestimation of noise in the time of speech activity. Sunitha et al (2007) [19] utilized DCT for normalizing the Least Mean Square for improving the speech quality used to implement in real time application. This algorithm is utilized to minimize the convergence range of the LMS for Sensory neural loss patients' applications. The characteristics of the DCT are ortho-normal, separable, and energy compaction and good signal decorrelator. In order to estimate this DCT-LMS algorithm SNR and eigenvalue ration computation parameters is used. Even though the DCT produces better outcomes, it does not separate frequencies when compared to other algorithms.

\section{INFERENCE FROM EXISTING SOLUTION}

This section comprises of the main disadvantage of speech enhancement for hearing aids

1. The main limitation of wiener filter is that the signal components coming from various directions, it concerns the overall performance of this method. The attained outcome of this method is not as much efficient.

2. The residual noise is one of the main drawbacks in speech enhancement method.

3. The main limitation of the beam former is a strong amplification of low frequency microphone noise components.

4. MWF-ITF needs the binaural unmasking effect for diverse speech and noise ITFs on a single frequency case of a speech quality improvement. However the binaural unmasking effect is not feasible in MWF-ITF and it is the key weakness of MWF-ITF.

5. The major weakness of this method is covariance matrix can be closely partial for small samples due to maximum likelihood.

6. The directional speech enhancement pattern has less performance than non-directional pattern while directional porter had more noise.

7. The hearing aid with directional speech enhancement pattern is highly perceptive to wind noise than a nondirectional pattern.

8. The major drawback of this method is an evaluation of echo time can be highly partial for small samples due to maximum likelihood.
9. The time domain filter is utilized to attain high speech quality, however the constructing a high quality filter is much difficult. This is the main limitation of time domain filter and another one is filter endures from the ripple.

10. Blind source separation is highly complicated to solve and computationally more costly.

11. The main limitation that it includes very narrow peaks in speech spectrum ensuing in overestimation of noise in the time of speech activity.

12. Even though the DCT produces better outcomes, it does not separate frequencies when compared to other algorithms.

\section{EXPERIMENTAL ANALYSIS}

This section discusses about the performance evaluation of various techniques with some parameters. In this work, the five well-known speech enhancement methods are analyzed and their performances are estimated through the performance metrics such as PSEQ and SNR. These five methods are mostly exploited to diminish the consequence of additive white Gaussian noise in the original speech signal in hearing aids. The methods used in the experiments incorporate Wiener filtering, beamformer, generalized singular value decomposition, transformations, and spectral subtraction technique. Here the AURORA database is exercised for an experimental estimation purpose and sample signals are obtained from the database to evaluate the performance of five approaches.

In the preprocessing method, the input speech signal is sampled and the time series signal is separated into many frames that are signified via a Hankel matrix. In this experiment the number of samples in each frame is going to be 600 . After the preprocessing various clean speech signals are at random selected from the database and followed by infected through various levels of white additive noise (from $0 \mathrm{~dB}$ to $15 \mathrm{~dB}$ ) are exercised for experimental assessment. After that the five speech enhancement approaches are executed on each noisy speech and accordingly the averaged SNR and PESQ results are illustrated in Fig. 1 and Fig. 2, correspondingly.

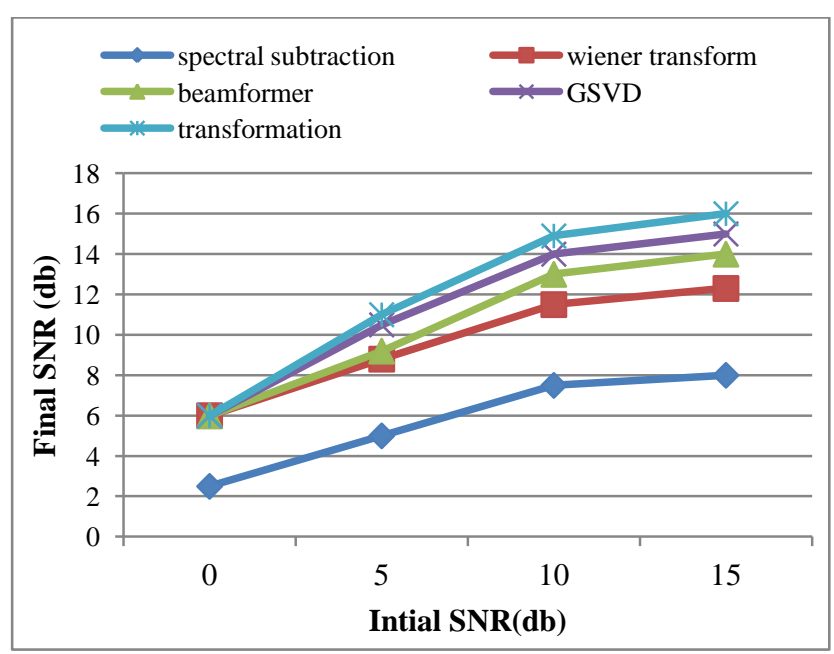

Figure 1: SNR results for white Gaussian noise case at varying SNR levels $(0,+5,+10$ and $+15 \mathrm{~dB})$ 


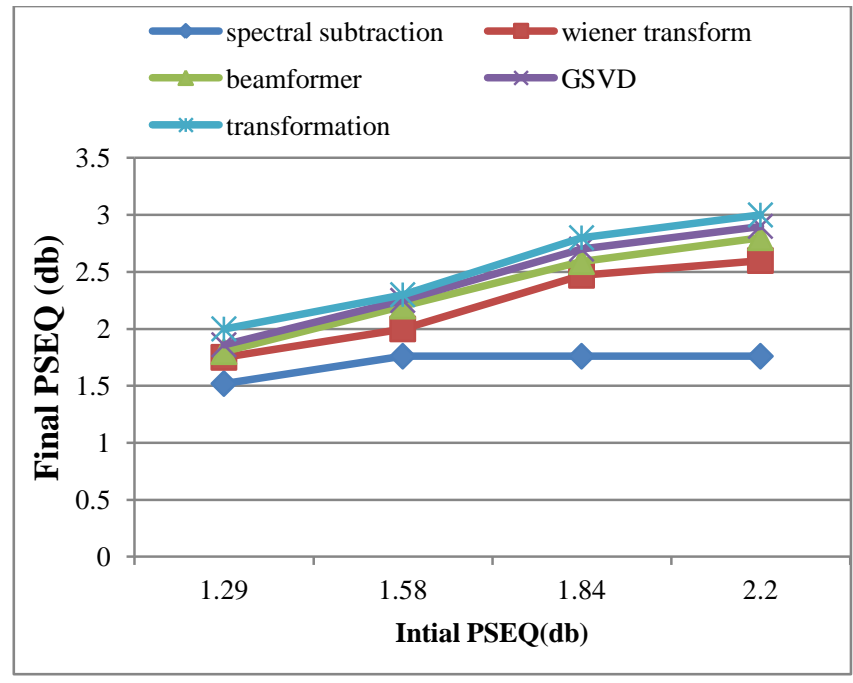

Figure 2: PESQ results for white Gaussian noise at varying $\mathrm{SNR}$ levels $(0,+5,+10$ and $+15 \mathrm{~dB})$

\section{CONCLUSION}

In this paper, many speech enhancement methods for hearing aids have been discussed. For the hearing impaired persons, speech enhancement algorithms in hearing aids are important due to the background noise present in the original speech signal. With the aim of improving the speech quality five different approaches are in general utilized to reduce background noise. The five different methods have been used here are Wiener filtering, beamformer, generalized singular value decomposition, transformations, and spectral subtraction technique. The advantages and disadvantages of these approaches are discussed in short. The performance of this algorithm is estimated using the performance metrics of SNR and PSEQ.

\section{REFERENCES}

[1] Dillon .H 2001, Hearing Aids. New York: Thieme Medical.

[2] Kulkarni P. N, Pandey P.C, and Jangamashetti .D.S 2012, "Binaural dichotic presentation to reduce the effects of spectral masking in moderate bilateral sensorineural hearing loss," International Journal of Audiology, Vol. 51, No. 4, pp. 334-344.

[3] Yang .J, Luo.F, and Nehorai .A 2003, "Spectral contrast enhancement: Algorithms and comparisons," Speech Communication, Vol. 39, No. 1-2, pp. 33-46.

[4] Kulkarni P. N, Pandey P. C, and Jangamashetti .D. S 2012, "Multi-band frequency compression for improving speech perception by listeners with moderate sensorineural hearing loss," Speech Communication Vol. 54, No. 3 pp. 341-350.

[5] Loizou .P.C 2006, "Speech processing in vocoder-centric cochlear implants," in A. R. Moller (ed.), Cochlear and Brainstem Implants, Adv. Otorhinolaryngol. Vol. 64, Basel: Karger, pp. 109-143.

[6] Junfeng Li, Shuichi Sakamoto, Satoshi Hongo, Masato Akagi and Yoiti Suzuki 2009,"Two-Stage Binaural Speech Enhancement With Wiener Filter Based On Equalization-Cancellation Model", IEEE Workshop on Applications of Signal Processing to Audio and Acoustics , ,pp. 133 - 136 ,October 18-21.
[7] Santosh K. Waddi, Prem C. Pandey, and Nitya Tiwari 2013, "Speech Enhancement Using Spectral Subtraction and Cascaded-Median Based Noise Estimation for Hearing Impaired Listeners" National Conference on Communications (NCC), pp: $1-5$.

[8] Heinrich W. Lollmann and Peter Vary 2012, "Beamformer For Driving Binaural Speech Enhancement" International Workshop on Acoustic Signal Enhancement, pp-0-4 , 4-6.

[9] Tim Van den Bogaert, Jan Wouters, Simon Doclo, Marc Moonen 2007, "Binaural Cue Preservation for Hearing Aids using an Interaural Transfer Function Multichannel Wiener Filter", IEEE International Conference on Acoustics Speech and Signal Processing, Vol.4 ,pp: 565 - 568.

[10] Ulrik Kjems and Jesper Jensen 2012, "Maximum Likelihood Based Noise Covariance Matrix Estimation For Multi-Microphone Speech Enhancement" European Signal Processing Conference (EUSIPCO 2012) Bucharest, Romania, pp:295-299, August 27 - 31.

[11] John Woodruff and DeLiang Wang 2011, "Directionality-Based Speech Enhancement For Hearing Aids" IEEE International Conference on Acoustics, Speech and Signal Processing (ICASSP), pp: 297 - 300, 22-27.

[12] HeinrichW. L"ollmann and Peter Vary 2009, "Low Delay Noise Reduction and Dereverberation for Hearing Aids" EURASIP Journal on Advances in Signal Processing Vol 2009, Article ID 437807, 9 pages.

[13] Klaus Reindl, Yuanhang Zheng, and Walter Kellermann 2010, "Speech Enhancement for Binaural Hearing Aids based on Blind Source Separation" 4th International Symposium on Communications, Control and Signal Processing (ISCCSP), pp: $1-6$.

[14] JEON Yu-yong, LEE Sang-min 2011, “A speech enhancement algorithm to reduce noise and compensate for partial masking effect" Journal of Central South University of Technology, Vol.18,No.4 pp.1121-1127.

[15] Young Woo Lee, Sang Min Lee, Yoon Sang Ji, Jong Shill Lee 2007, Young Joon Chee, Sung Hwa Hong, Sun I. Kim , In Young Kim, "An Efficient Speech Enhancement Algorithm for Digital Hearing Aids Based on Modified Spectral Subtraction and Companding", Journal IEICE Transactions on Fundamentals of Electronics, Communications and Computer Sciences, Volume E90-A Issue 8,pp: 1628-1635.

[16] Simon Doclo, and Marc Moonen 2002, "GSVD-Based Optimal Filtering for Single and Multimicrophone Speech Enhancement" IEEE Transactions on Signal Processing, Vol. 50, No. 9, pp: 2230 - 2244

[17] Dr. (Smt). S.D. Apte , Shridhar 2009,"Speech Enhancement in Hearing Aids Using Conjugate Symmetry of DFT and SNR-Perception Models" International Journal of Recent Trends in Engineering, Vol. 2, No. 5, pp:346-351.

[18] Sunitha. S.L., and V. Udayashankara 2007,"Fast Factored DCT-LMS Speech Enhancement for Performance Enhancement of Digital Hearing Aid" International Journal of Medical, Pharmaceutical Science and Engineering Vol.1 No.10, pp:568-572. 